\title{
A Holistic Design Perspective on Media Capturing and Reliving
}

\author{
Mendel Broekhuijsen ${ }^{1,2}$ \\ m.j.broekhuijsen@tue.nl \\ ${ }^{1}$ Eindhoven University of \\ Technology \\ Dept. of Industrial Design \\ Eindhoven, the Netherlands
}

\author{
Ine Mols ${ }^{1,2}$ \\ i.mols@tue.nl \\ ${ }^{2}$ University of Technology \\ Sydney \\ Faculty of Engineering and \\ Information Technology \\ Sydney, Australia
}

\author{
Elise van den Hoven ${ }^{2,1,3,4}$ \\ elise.vandenhoven@uts.edu.au \\ ${ }^{3}$ ARC Centre of Excellence in \\ Cognition and its Disorders
}
${ }^{4}$ DJCAD University of Dundee Dundee, UK

\begin{abstract}
People capture far more media than they are able to relive. In this paper we identify the discrepancy between media capturing and media reliving from a design perspective. We propose a holistic perspective, that invites designers of media experiences to considering all three interdependent aspects of the media process: media capturing interaction, specific media, and media reliving interaction. By adopting this view, we aim to ensure that the media that is captured will be both necessary and appropriate for the intended reliving experience. We illustrate our perspective with three design concepts. Finally, in the discussion we present several topics related to media capturing and reliving.
\end{abstract}

\section{Author Keywords}

Interaction Design; Digital Media; Design for Remembering; User Experience; Photography

\section{ACM Classification Keywords}

H5.m. Information interfaces and presentation (e.g., HCI): Miscellaneous.

\section{INTRODUCTION}

Since the introduction of digital capturing devices such as compact cameras, smartphones and DSLR cameras the accumulation of media has increased exponentially. People can create vast quantities of media, without having to worry about storing them. But an often heard comment from photography enthusiasts is that they do not take the time to view the photos that they have taken, despite their intentions. People can have a variety of purposes in mind for media capturing, including formative, communicative, experiential or remembering purposes (Van Dijck, 2008). We are especially interested in media that is captured for remembering purposes. Photos and other media have the potential to support remembering the past by serving as cues for autobiographical memories (van den Hoven,

Permission to make digital or hard copies of all or part of this work for personal or classroom use is granted without fee provided that copies are not made or distributed for profit or commercial advantage and that copies bear this notice and the full citation on the first page. Copyrights for components of this work owned by others than ACM must be honored. Abstracting with credit is permitted. To copy otherwise, or republish, to post on servers or to redistribute to lists, requires prior specific permission and/or a fee. Request permissions from Permissions@acm.org.

$\mathrm{OzCHI}$ '16, November 29-December 02, 2016, Launceston, TAS, Australia
2014), and within HCI research there is a growing interest in design for remembering (Van den Hoven, Sas \& Whittaker, 2012). However, in our view, most design concepts do not use media to their full (remembering) potential. We see two trends in domestic media technology that influence this. The first trend is a general over-presence of technologies that offer media capturing, but that do not specifically address to what purpose the media is captured and thus what should be done with them. The second trend is that tools intended to support media reliving generally fail to address what kind of media types should be captured to best support the desired reliving experience.

In this paper we identify the discrepancy between media capturing and media reliving from a design perspective, and propose an alternative, more holistic perspective. We illustrate this perspective with three design concepts and present several topics for discussion.

\section{RELATED WORK}

There are many interesting examples of interactive systems that have (re)designed a specific aspect of the media process, both from research and industry. Some systems focus on novel technologies of capturing (e.g. Hakansson et al. 2006; Niforatos et al. 2014; Narrative, getnarrative.com; Google Glass, google.com/glass/start/). Others focus on new media types (e.g. Frohlich, 2004; Dib, Petrelli \& Whittaker, 2010; Güldenpfennig et al. 2012b; Live Photos within Apple's iOS, apple.com/ios/photos/). Other concepts create engaging reliving interactions (e.g. Uriu et al. 2009; Meerbeek et al. 2010; Odom et al. 2012; Golsteijn \& van den Hoven, 2013; Kuchelmeister \& Bennet, 2014). An extensive review of all the examples goes beyond the scope of this paper. Instead, we discuss three examples in more detail that illustrate our main observation from literature: redesigns of media technology often focus on one of the above mentioned aspects, while it remains unclear how this affects other media aspects.

Cueb (Golsteijn \& Van den Hoven, 2013) is a redesign of media reliving, focussed on bonding between parents and teenagers. The interactive cubes use meta-data to present relevant combinations of photos to spark a conversation. However, it remains unclear how the media will be enriched with the required metadata (e.g. time, location, people) to allow photos to be appropriately filtered. In other words, the envisioned reliving scenario dictates the 
availability of media with high-quality meta data, while that information is rarely present in current collections.

The project Context Photography (Hakansson et al. 2006) redesigned the media type, by introducing photographs that are visually influenced by contextual factors. The evaluation shows how the interaction influences the way people experience media capturing. We believe that the experience of reliving these media is also different from traditional photos, because of the more abstract content. However, the concept does not include suggestions for reliving beyond the traditional screen-based viewing of content. As a result, the media might not be used to its full potential.

The design of Behind the Camera (Güldenpfennig et al. 2012a) does provide suggestions in their evaluation for both capturing and reliving of novel media. The concept uses both the front facing and the rear camera on a smartphone to simultaneously capture photos from two sides. The resulting sets of photos can be viewed as double sided prints, or as sets for a memory-like matchmaking game. These exercises show an interesting direction for playful reliving, connected to the playful capturing interaction. Current commercially available applications for digital photography typically lack such connections. They treat all photos like they are the same, and lack the connection between with the means of capturing. One could argue that selfies taken with a smartphone need to be relived differently from landscape photos taken with a DSLR camera.

Based on these concepts and other similar work, we have observed that several concepts have quite a narrow focus. Either the capturing is redesigned, leaving questions unanswered on how to manage and relive the resulting media. In other cases, a specific reliving experience is redesigned, with less details on how the required media is created, collected and managed. To be able to address this discrepancy between capturing and reliving, we propose a more holistic view on the media process.

\section{HOLISTIC MEDIA PERSPECTIVE}

The discrepancy between the means to capture media and the technologies for reliving can be addressed if the relations between both are considered. We believe that designers should considering both capturing and reliving as two parts of the same, holistic process. Various researchers have presented models that describe the practices and related technologies, to inform design. Such as the Photowork model (Kirk et al. 2006) and the holistic model of purposive PhotoUse (Broekhuijsen et al., in press). We contribute to this area by presenting a simplified perspective, pointing out the various aspects that we believe should be considered in parallel, not in isolation. Figure 1 illustrates our point of view, and describes the three interdependent aspects of the media process: media capturing interaction, specific media and media reliving interaction. The capturing interaction determines specific media; specific media determines what kind of reliving interactions are possible (the blue arrows in Figure 1); reliving interactions require a specific media type; specific media type requires a certain capturing interaction (the green arrows in Figure 1). To give an example: a novel immersive reliving experience could consist of a room with $360^{\circ}$ projections of visited holiday locations. This requires a specific media type: $360^{\circ}$ photos. In turn, this media needs to be captured, which can be done, which can be done using tools like the Ricoh Theta camera (theta360.com/). In this way the media capturing, media type and reliving interaction are directly connected.

Our proposed change of perspective does not dictate where designers should start with (re)designing novel media technologies to support remembering. Instead, each aspect can be the starting point for a (re)design, as long as the consequences for the other aspects are considered. Connections between the different aspects can be made on multiple levels. First of all, on a very practical level (such as the $360^{\circ}$ example) but also on levels of experience and intention. We will illustrate these different connections in the next section. By adopting this view, we aim to ensure that the media that is captured will be both necessary and appropriate for the intended reliving experience.

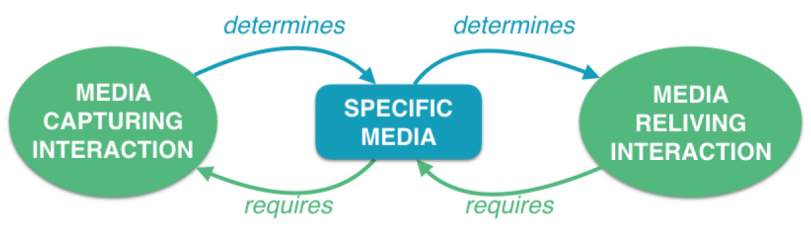

Figure 1: Illustration of the holistic perspective on designing novel media experiences

\section{DESIGN EXAMPLES}

To further illustrate our holistic perspective on the media experience process, we present three design concepts. Each concept was developed to redesign the media experience during a specific day out. Each concept has its main focus on one of the three media aspects, but deliberately used the holistic perspective during the entire design process. For each concept we describe the main design aspects and the relation to the holistic media perspective.

\section{Playful media creation: Zoovenir}

The design of Zoovenir was inspired by observing families at a local zoo, which revealed a disconnection between the behaviour and interest of young children and the capturing behaviour of parents. E.g., while a father was explaining something about an animal, his child was more interested in some colourful electric wiring on the ground. Zoovenir was designed to capture the child's perspective.

Through a capturing binocular, bought at the zoo's entrance, young children (ages four to eight) fulfil a quest and receive prompts to explore the zoo (Figure 2). These open-ended prompts, ask to capture "something red" or "something round", allowing for interpretation based on the child's perspective and interest. While the child focuses on completing the quest, media is created of each accomplished prompt. The photos are saved on the dedicated binocular, together with metadata, such as the child's perspective and viewing angle. Reliving is designed to be similarly playful, when occurring years 
later as a way of getting to know a younger version of oneself. When looking through the binocular the user sees just a small fragment that was captured. To reveal the full picture and the context in which it was taken, they have to search for the perspective and angle that the picture was taken at. As such, reliving becomes more than reviewing the media, it recreates the playful experience of the time.

\section{Connection to the holistic perspective}

Zoovenir was designed with the capturing interaction as starting point. From this starting point the other aspects were considered both on a practical level and to match a specific experience. The child's playful experience of exploring is integrated into the capturing prompts, which is also translated in the design of the physical and digital media, and in the interaction of reliving. The coupling between the different aspects is very explicit in this case, since the device, interaction and experience are designed to be similar in all stages.

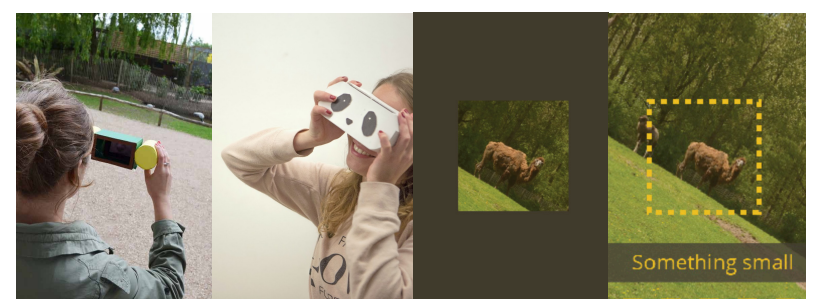

Figure 2: Zoovenir. f.l.t.r. capturing interaction; reliving interaction; media partly revealed; media fully revealed after finding the original capturing angle

\section{Contextualised media reliving: Objekt}

The majority of digital photo collections are stored in large bulk on a hard drive hidden away and rarely accessed or relived (Petrelli \& Whittaker, 2010). In contrast, our physical memorabilia are often on display in our homes, as cues for remembering, but also to display a personal identity, to serve as conversation starters or to make a house feel like home (Petrelli \& Whittaker, 2010). The design of Objekt was inspired by this discrepancy between physical and digital. The reliving of digital photos was redesigned with the aim to make the experience more accessible, social and tangible. Similar to the designs by Van den Hoven \& Eggen (2003) and Nunes et al. (2008), Objekt connects digital photos to objects or locations within the home. Using small, inexpensive RFID stickers, objects can be tagged to connect them to digital content. These augmented physical representations (a combination of a tangible object with associated digital media) became the new media type central to the design. In contrast to the other two concepts, Objekt allows for the creation of these hybrid media while on the go. The concept leverages the advantages of smartphones' capabilities through a specific application that enables users to make a direct coupling with an object, right before capturing. The result is a photo collection that is distributed throughout the house, allowing for contextualised reliving (Figure 3).

The simple scenario of connecting digital photos to objects sparks several interesting possibilities because the coupling can be done in multiple ways. Connections can be made based on the 'content' of the memory, for instance by connecting photos from a trip to unique souvenirs. The connection could also be made based on an envisioned reliving scenario, stimulating the user to think about when and where s/he would like to view the picture and relive the memory, before taking the photo. This might lead to e.g. connecting specific photos to a coffee pot, which would allow reliving these memories when someone visits for coffee.

\section{Connection to the holistic perspective}

Objekt was designed from the goal to make digital media easier to access for satisfying reliving experiences. After conceptualising how digital media could be relived through connections with the physical, a corresponding media type and capturing practices were designed to enable this reliving. This concept also illustrates that if the designers explicitly consider the holistic media perspective, connections between capturing and reliving can also be made by the users. We will come back to the challenge of user intention in the discussion.

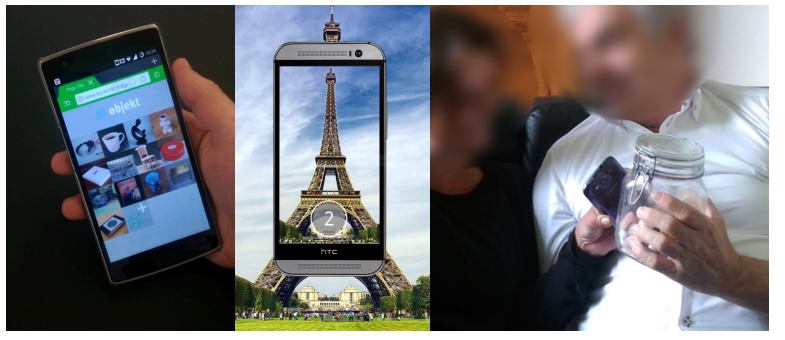

Figure 3: Objekt. f.l.t.r. Choosing object to link the next photo to; photo capturing; media reliving at home by using objects to retrieve the photos

\section{Personalizing Public Media: Festory}

Media capturing during festivals and music concerts is more and more criticised, both audience and artists express being frustrated by the number of phones and cameras that obstruct direct contact. However, people often feel the desire to capture these memorable experiences. Festory creates unique personal media from a festival without interrupting the experience itself. The concept consists of a smart bracelet, a media archive, and an online platform (Figure 3). To allow users to create a personal aftermovie, the Festory system uses professional video material, which is of much higher quality than amateur material. The movie is personalised by combining movement data with locations. For example, one individual's aftermovie will include the ballad during which the crowd was waving from left to right and the encore to which everybody was jumping around. During the festival, the required data is collected unobtrusively and effortless through the bracelet. The Festory algorithm collects relevant video fragments from moments of high enthusiasm and combines these into a personal aftermovie. The aftermovies are shared and viewed on an online platform, allowing interaction with other visitors.

\section{Connection to the holistic perspective}

In our view, the novel aspect of Festory is mainly found In the new media type. The aftermovies can be seen as public-private media, leveraging the amount and quality of publicliy available media but filtering it based on personal aspects. Festory redesigns the media process by focussing on the user not capturing media, or at least, not 
investing effort in media capturing. The aspects of media capturing and media type are strongly linked in this concept. However, the reliving experience could be further redesigned to better connect to designed elements of limited effort and public-private media.

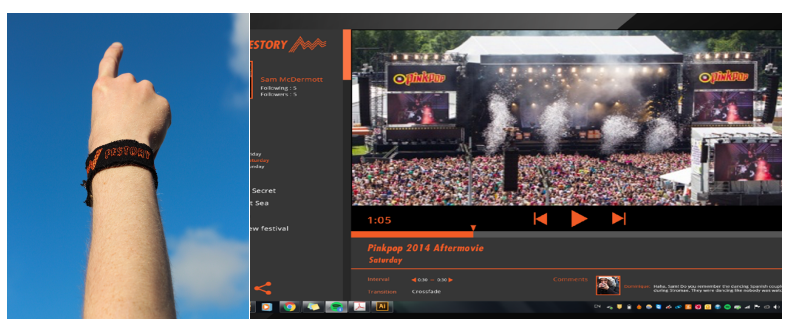

Figure 4: Festory. Automated capturing interaction using bracelet (left); reliving the personalized aftermovie (right)

\section{DISCUSSION}

With our holistic media perspective, we hope to inspire design of engaging media experiences with strong connections between the different aspects. Media is captured for specific reliving purposes and in turn, reliving scenarios are supported by capturing suitable media. The diversity of the concepts in terms of context, starting point and media type show the flexibility of the approach. However, the perspective also sparks several topics for discussion.

\section{Model Validation}

The holistic perspective as described in this paper is in an early stage of development. In our view, the perspective can contribute to design research as generative intermediate level design knowledge (Höök, K., \& Löwgren, J. 2012). The perspective can contribute to generating ideas, concepts and directions that are not only novel in terms of user experience, but are also better suited for the evolving media practices. The student project that resulted in the described concepts, strengthened our believe in the holistic perspective as a valuable vantage point for designing media experiences. We valued the holistic perspective for its ability to assist the students and us to assess the completeness of the designs that were developed during the project, providing concrete pointers for improvements. To further develop the perspective towards a useful tool in itself, both the perspective's contribution for generating ideas and the resulting concepts should be evaluated more systematically.

\section{Dedicated or Multi-Purpose Devices}

One of the trade-offs coming forth from the holistic perspective, is between developing devices that are dedicated for specific capturing practices, and developing a single tool that can support multiple practices. Zoovenir illustrates that a dedicated concept for a specific context can create engaging experiences with strong connections between the different aspects. However, having a dedicated device for every type of memorable experience might not be desirable. In a design process, such dedicated tools could function as design exercise to challenge the connections between the three aspects, which can later be translated to more versatile concepts. Objekt shows that even more versatile capturing concepts can have explicit connections between the aspects, because the effects on all aspects are still considered.

\section{Media Quantity}

The holistic approach can also be interesting to reframe the earlier mentioned problems with photo curation. Some argue that the huge quantities of media people own is the reason that they do not look at them anymore. With the holistic perspective the problem can be framed in multiple ways. To make stronger connection between the different aspects one could limit the amount of media captured (e.g. Niforatos et al. 2014). In contrast, one can also consider how to create effective reliving strategies for high quantity of media. Few examples are making good use of the abundance of media, such as Kuchelmeister \& Bennet (2014), who displayed photos taken from a lifelogging device on a projected landscape route. In general, high quantity of media amplifies the discrepancy between capturing and reliving. The holistic design perspective allows designers to approach such a challenge in multiple ways and to make connections between the media aspects on different levels.

\section{Intention to Remember}

Throughout this paper we emphasise that it is a designer's responsibility to consider the impact of novel media technologies on others aspects of the media process. In the case of Zoovenir, the users do not explicitly choose how to remember the experience but instead the responsibility of capturing appropriate media for reexperiencing and remembering is integrated into the design. However, in some cases, part of the responsibility to connect media capturing with intended reliving can be shared with the users. Objekt more explicitly gives the user the responsibility to consider how media will be relived. Because the user has to choose a context for the digital media, a prospective reliving experience needs to be considered. In other words, a designer can take the responsibility to consider the consequences in designing media capturing and reliving, while part of this responsibility can be explicitly entrusted to the user.

However, it is debatable whether people are able to estimate what media to develop for future remembering. A direct connection between capturing and reliving also endangers room to revalue photographic material, while the perceived value of both memories and media can change over time (Van House et al. 2005, Zhang et al. 2014). Further research is needed to get a better grasp of the suitable level of user responsibility for envisioning reliving, and capturing suitable media.

\section{CONCLUSION}

Media can have high value to remember personal experiences, however current technology does not support capturing media that are used to their full potential. With the holistic design perspective, we aim to spark new design opportunities for engaging media concepts. The perspective proposes to create strong connections between media capturing, media type and media reliving, in order to address the discrepancy between these aspects.

\section{ACKNOWLEDGMENTS}

This research was supported by STW VIDI grant number 016.128.303 of The Netherlands Organization for 
Scientific Research (NWO), awarded to Elise van den Hoven. We thank the students and their coaches for their contributions: Nicky Pronk, Daborah Pulles, Danielle Ramp \& Enna Rizvic, coached by Fiona Jongejans (Zoovenir); Thomas Bouwman \& Oscar van Beek, coached by Mendel Broekhuijsen (Objekt); Dominique Fürst, coached by Julie Hornix (Festory). We also thank Panos Markopoulos and Berry Eggen for their advice on the paper.

\section{REFERENCES}

Broekhuijsen, M., Hoven, E. van den, and Markopoulos, P. In Press. From PhotoWork to PhotoUse: Exploring Personal Digital Photo Activities. Behaviour \& Information Technology. Taylor \& Francis.

Dib, L., Petrelli, D., and Whittaker, S. 2010. Sonic souvenirs: exploring the paradoxes of recorded sound for family remembering. In: Proc. of $C S C W^{\prime} 10$. ACM, New York, NY, USA, 391-400.

Dijck, J. van. 2008. Digital Photography: Communication, Identity, Memory. Visual Communication 7, 1: 57-76.

Frohlich, D. 2004. Audiophotography: Bringing photos to life with sounds. Springer Science \& Business Media.

Golsteijn, C. and Hoven, E. van den. 2013. Facilitating parent-teenager communication through interactive photo cubes. Personal and Ubiquitous Computing 17, 2: 273-286.

Güldenpfennig, F., Reitberger, W., and Fitzpatrick, G. 2012a. Of Unkempt Hair, Dirty Shirts and Smiling Faces: Capturing Behind the Mobile Camera. In Proc. of NordiCHI'12. ACM, NY, USA, 298-307.

Güldenpfennig, F., Reitberger, W., \& Fitzpatrick, G. 2012b. Capturing rich media through media objects on smartphones. In Proc. of OzChi'12. ACM, NY, USA, 180-183.

Hakansson, M., Gaye, L., Ljungblad, S., and Holmquist, L.E. 2006. More than meets the eye: an exploratory study of context photography. In Proc. NordiCHI'06. ACM, NY, USA, 262-271.

Höök, K., \& Löwgren, J. 2012. Strong concepts: Intermediate-level knowledge in interaction design research. ACM Transactions on Computer-Human Interaction (TOCHI), 19(3), 23.

Hoven, E. van den, and Eggen, B. 2003. Digital Photo Browsing with Souvenirs. In Proc. of Interact 2003. 1000-1003.
Hoven, E. van den, Sas, C., and Whittaker, S. 2012. Introduction to this special issue on designing for personal memories: past, present, and future. HumanComputer Interaction 27, 1-2: 1-12.

Hoven, E. van den. 2014. A future-proof past: Designing for remembering experiences. Memory Studies 7, 3: 370-384.

Kirk, D., Sellen, A., Rother, C., and Wood, K. 2006. Understanding Photowork. In Proc. of CHI'06. ACM, NY, USA, 761-770.

Kuchelmeister, V. and Bennet, J. 2014. The Amnesia Atlas. An immersive SenseCam interface as memoryprosthesis. In Proc. of VSMM 2014, 217-222. IEEE.

Meerbeek, B., Bingley, P., Rijnen, W. and Hoven, E. van den. 2010. Pipet: a design concept supporting photo sharing. In Proc of NordiCHI '10. ACM, New York, NY, USA, 335-342.

Nunes, M., Greenberg, S., and Neustaedter, C. 2008. Sharing digital photographs in the home through physical mementos, souvenirs, and keepsakes. In Proc. of DIS '08. ACM, New York, NY, USA, 250-260

Niforatos, E., Langheinrich, M. and Bexheti, A. 2014. My good old kodak. UbiComp '14 Adjunct. ACM, NY, USA, 1355-1360.

Odom, W., Selby, W., Sellen, W., Kirk, D.S., Banks, R., and Regan, T. 2012. Photobox: on the design of a slow technology. ACM, NY, USA, 665-668.

Petrelli, D. and Whittaker, S. 2010. Family memories in the home: contrasting physical and digital mementos. Personal and Ubiquitous Computing, 14(2), 153-169.

Uriu, D., Shiratori, N., Hashimoto, S., Ishibashi, S., and Okude, N. 2009. CaraClock - an interactive photo viewer designed for family memories. In: CHI '09 Extended Abstracts. ACM, NY, USA. 3205-3210.

Van House N.A., Davis, M., Ames, M. and Finn, M. 2005. The uses of personal networked digital imaging: an empirical study of cameraphone photos and sharing. CHI'05 Extended Abstracts. ACM, NY, USA. 18531856.

Zhang, T., Kim, T., Brooks A.W., Gino, F., and Norton, M.I. 2014. A "present" for the future: the unexpected value of rediscovery. Psychological science 25, 10: 1851-1860. 